\title{
On the Study of Sustainability and Outage of SWIPT-Enabled Wireless Communications
}

Luo, Y., Luo, C., Min, G., Parr, G., \& McClean, S. I. (2021). On the Study of Sustainability and Outage of SWIPTEnabled Wireless Communications. IEEE Journal of Selected Topics in Signal Processing, 15(5), 1159-1168. https://doi.org/10.1109/JSTSP.2021.3092136

Link to publication record in Ulster University Research Portal

Published in:

IEEE Journal of Selected Topics in Signal Processing

Publication Status:

Published (in print/issue): 01/08/2021

DOI:

10.1109/JSTSP.2021.3092136

\section{Document Version}

Author Accepted version

\section{General rights}

Copyright for the publications made accessible via Ulster University's Research Portal is retained by the author(s) and / or other copyright owners and it is a condition of accessing these publications that users recognise and abide by the legal requirements associated with these rights.

\section{Take down policy}

The Research Portal is Ulster University's institutional repository that provides access to Ulster's research outputs. Every effort has been made to ensure that content in the Research Portal does not infringe any person's rights, or applicable UK laws. If you discover content in the Research Portal that you believe breaches copyright or violates any law, please contact pure-support@ulster.ac.uk. 


\title{
On the Study of Sustainability and Outage of SWIPT-Enabled Wireless Communications
}

\author{
Yang Luo, Chunbo Luo, Geyong Min, Gerard Parr, Sally McClean
}

\begin{abstract}
Wireless power transfer technologies such as simultaneous wireless information and power transfer (SWIPT) have shown significant potentials to revolutionise the design of future wireless communication systems. When the only energy source is from the wireless signals that are mainly intended for information communications, the sustainability and outage performance of SWIPT systems become critical factors in theoretical evaluation and practical applications. This paper firstly models the energy harvesting and energy consumption of the power splitting protocol based SWIPT systems to investigate the general sustainability condition. We further model the power and information transfer outage probabilities using Markov Chain, which are unique for SWIPT systems since they both could cause communication outage. We further demonstrate how to apply the closed-form expression of the outage to optimise the key parameter of splitting ratio for SWIPT systems. Hardware and numerical experiments demonstrate the validity of the proposed model and outage analysis, and confirm the effectiveness of the solution to calculate the optimal splitting ratios under different signal and channel conditions.
\end{abstract}

Index Terms-Wireless power transfer, SWIPT, outage, wireless communications

\section{INTRODUCTION}

Wireless power transfer (WPT) could shift the paradigm of future wireless communications due to its unprecedented benefits to lift the dependency of communication systems on power supply infrastructure and enable higher freedom for both design and deployment of communication networks [1]-[4]. While other energy harvesting approaches such as kinetic and solar energy harvesters can derive energy from the ambient environment sources, the availability and density of them are uncontrollable [5]. Simultaneous wireless information and power transfer (SWIPT) offers a potentially controllable way to top up energy storage by optimising the two fundamental resources of wireless signals including energy and information, showing significant potentials in energy-constrained scenarios such as telemedicine, remote sensing, smart cities, as well as general wireless communication systems [6]-[9].

Y. Luo is with the School of Information and Communication Engineering, University of Electronic Science and Technology of China, Chengdu, 611731 China (e-mail: luoyang@uestc.edu.cn).

C. Luo and G. Min are with the College of Engineering, Mathematics and Physical Sciences, University of Exeter, Exeter, EX4 4QF, UK (e-mail: \{c.luo,g.min\}@exeter.ac.uk).

G. Parr was with School of Computing Sciences, University of East Anglia, Norwich, NR4 7TJ, UK (e-mail: g.parr@uea.ac.uk).

S. McClean was with School of Computing, University of Ulster, Newtownabbey, BT37 0QB, UK (e-mail: si.mcclean@ulster.ac.uk).
Existing studies on energy harvesting from wireless signals have established the theoretical ground for SWIPT, where two typical protocols could be adopted to split the power for either energy harvest or information transfer: time switching (e.g., [10], [11]) and power splitting (PS) (e.g., [12], [13]). Considering the competition of these two tasks for the same resource and the low energy-carrying capability of radio frequency (RF) signals, PS based SWIPT is gaining increased attention due to its higher spectral efficiency [13], [14]. Promising research work on SWIPT has addressed some key issues including, novel harvesting circuits and antenna design (such as innovative rectifiers and multi-antenna systems [12], [15]-[17]), communication and cooperation protocols (such as routing and scheduling methods [3], [18]), and harvesting and communication strategies ( [5], [14]).

For SWIPT systems, the propagation loss and low power carrying capability of RF signals could lead to potentially frequent energy depletion and communication failure [2], [7]. It becomes essential to study the outage performance of such systems in order to investigate their sustainability, e.g., [5], [13]. Specifically, Lu et al. [13] considered the scenario where SWIPT has the opportunity to harvest energy from both the intended transmitter and ambient signals. Peng et al. established the boundary conditions for achieving an energy neutral state within a wireless sensor network [19]. Ding et al. explored the important theoretical tool of outage to analyse the performance of SWIPT systems [20]. Existing research work has established the valuable foundation for SWIPT, but they often adopt theoretical models and parameters without underpinning from realworld perspectives. Furthermore, most outage analysis focuses on information transmission and neglects another important outage factor caused by insufficient power from the harvester, which is often unique in SWIPT systems.

This paper firstly introduces the energy harvesting and power consumption models for a wireless communication system and then evaluates their relationship by comparing the average income and consumption to establish the sustainability condition. Since wireless signals usually suffer from channel effects such as fading, we model the problem using Markov Chains and calculate the power and information outage probabilities. We thoroughly investigate the short-term and long-term performance under different conditions, e.g. ambient power harvesting and storage in battery/supercapacitor, transceiver distance, and power splitting factors, derive a closed-form for the joint power and information outage, and present a low complexity 
approach to calculate the power splitting ratio, given the different parameters such as transmitting power, fading, and harvesting efficiency. We further evaluate the sustainability model and the proposed solution using numerical and hardware experiments, which confirm the performance of the proposed approaches. The key contributions of this paper are summarised below:

- Modelling the sustainability of SWIPT systems. Unlike most theoretical modelling work of SWIPT systems, we study the sustainability of SWIPT based on practical system parameters from both the energy harvesting and consumption perspectives. Hardware experiments confirm the effectiveness of such a model.

- Outage analysis of SWIPT systems. In addition to information outage, this paper studies the outage performance of SWIPT systems by analysing both the power and information impacts that are unique for SWIPT. The two outage probabilities are integrated into one joint closed form, allowing optimisation on the key relevant parameters such as power splitting ratio, transmitting power, harvesting efficiency etc.

- Optimal power splitting. We propose a low complexity approach to find the optimal power splitting ratio that leads to minimum outage based on the closed form expression. Numerical experiments confirm that the optimal values under varied system and channel conditions can always be calculated using this approach.

The remainder of the paper is as follows: Section II introduces the system model and energy consumption model, and proposes the sustainability condition for SWIPT. Section III investigates the power and information outage probability. Section IV provides hardware and numerical experiments to validate the sustainability condition and analyse the outage performance. Finally, Section V concludes this paper and discusses some promising future directions.

\section{System Model And Sustainability of SWIPT SYSTEMS}

A typical SWIPT system includes both the transmitting and receiving RF chains, a power splitter, an energy harvesting module and a storage module. This paper studies the impacts of all these key modules (The main system parameters are listed in Table I.). The power splitting factor $\theta_{s}$ defines the ratio of RF power being used for information transmission. If a wireless node has a rechargeable energy storage unit such as a battery or supercapacitor, the harvested energy from the transmitter and ambient signals will be stored in this unit and then used to power the signal processing circuit and RF chain.

A typical SWIPT system can be modelled as follows [20],

$$
y_{s}=\sqrt{\theta_{s}}\left(\frac{\sqrt{P} h x_{s}}{\sqrt{1+d^{\alpha}}}+n_{a}\right)+n_{p},
$$

where $y_{s}$ is the received signal after power splitting, $P$ denotes the transmitting power from the source, e.g. a base

\begin{tabular}{|c|c|}
\hline $\begin{array}{l}\text { Para- } \\
\text { meters }\end{array}$ & Description and exemplar values \\
\hline$P$ & Source transmitting power $(1 W)$ \\
\hline$\theta_{s}$ & Power splitting factor $(0.5)$ \\
\hline$\lambda$ & Average channel gain (1) \\
\hline$d$ & Transceiver distance $(3.8 m)$ \\
\hline$\alpha$ & Path loss factor (2) \\
\hline$\beta$ & Battery conversion efficiency (0.9) \\
\hline$\eta$ & Harvesting efficiency (1) \\
\hline$T_{s}$ & $\begin{array}{l}\text { Symbol duration } \\
\text { (for a BPSK symbol: } 1 /(256 K b p s) \text { ) }\end{array}$ \\
\hline$R$ & Data rate per $\mathrm{Hz}(1 b i t / H z / s)$ \\
\hline$E_{\text {elec }}$ & $\begin{array}{l}\text { Transmitting/receiving electronics energy } \\
\text { consumption per one bit }(50 \mathrm{~nJ} / \text { bit })\end{array}$ \\
\hline$E_{a m p}$ & Energy dissemination rate $\left(100 \mathrm{pJ} / \mathrm{bit} / \mathrm{m}^{2}\right)$ \\
\hline$I_{0}$ & Zero-th order of the first type Bessel function \\
\hline$S_{d B}$ & Ratio of direct and indirect energy in $\mathrm{dB}$ \\
\hline$\rho$ & Transmitting signal to noise ratio (SNR) \\
\hline
\end{tabular}

TABLE I: System parameters.

station or a wireless router, $h$ denotes the channel coefficient from the transmitter to the receiver, which could be modelled as a Rician distribution in most power harvesting scenarios. Because non line-of-sight (NLOS) propagation significantly attenuates the power of a wireless signal, the received power is much lower than LOS scenarios, for which Rician distribution provides more accurate modelling. $x_{s}$ is the source signal with unit power, $d$ is the distance between the transmitter and the receiver, and $\alpha$ is the path loss factor. The transmitting signal to noise ratio (SNR) is denoted by $\rho=P / \sigma_{p}^{2}$. The received signals are subject to two main types of noise: the antenna unit noise $n_{a}$ and signal processing unit noise $n_{p}$, modelled by Gaussian Distributions $N\left(0, \sigma_{a}^{2}\right)$ and $N\left(0, \sigma_{p}^{2}\right)$, respectively.

The harvested energy from wireless signals can be expressed as follows

$$
E_{s}=\eta\left(1-\theta_{s}\right)\left(\frac{P|h|^{2}}{1+d^{\alpha}}+\sigma_{a}^{2}\right) T_{s},
$$

where $\eta$ denotes the harvesting efficiency [12], [21], and $T_{s}$ is the symbol duration. Since $\sigma_{a}^{2}$ is usually very small, it is assumed to be 0 according to [10].

The stored energy at the battery or supercapacitor can be modelled as follows, subject to a conversion loss,

$$
E_{b}=\beta E_{s}=\beta \eta\left(1-\theta_{s}\right) P T_{s}\left(\frac{|h|^{2}}{1+d^{\alpha}}\right),
$$

where $\beta$ is the conversion efficiency factor. According to [22]-[24], for Lithium-ion batteries and supercapacitors, $\beta$ usually ranges from 0.9 to 0.95 .

\section{A. Energy consumption modelling}

We model the energy consumption of a wireless node according to [25], [26], consisting of two main parts: the circuit power consumption and the propagated power. In detail, the energy consumed at the circuit for receiving and 
transmitting one bit is $E_{\text {elec }}(n J / b i t)$, and the disseminated power is $d^{2} E_{a m p}$, where $E_{a m p}$ denotes the energy dissipating factor for maintaining an acceptable SNR level. This model also considers the energy consumption at the computing modules, denoted as $E_{\text {comp }}(n J / b i t)$. Therefore, the total energy required by a node to complete a communication session, e.g. receiving and transmitting a message of $N$ bits, can be approximated as follows:

$$
E_{c}=2 N E_{\text {elec }}+N d^{2} E_{a m p}+E_{\text {comp }}
$$

where the transmitter and receiver both consume $N E_{\text {elec }}$ energy, respectively. According to [25], [26], typical values of $E_{\text {elec }}$ and $E_{a m p}$ are $50 \mathrm{~nJ} / \mathrm{bit}$ and $100 \mathrm{pJ} / \mathrm{bit} / \mathrm{m}^{2}$, respectively, which are also used in the experiments of this paper. According to a recent study [27], the power consumption of the computing unit in a new design consumes $8 \%$ of the total circuit energy consumption, which is equivalent to $8.7 \% N E_{\text {elec }}$. It is expected that the exact consumption would decrease in future low-power systems.

\section{B. Sustainability of SWIPT systems}

To design a wireless communication system that relies on SWIPT based power harvesting, it is important to study its sustainability and identify the boundary condition to achieve sustainable communications. We thus propose the following definition:

Proposition 2.1: A SWIPT system is sustainable if $\mathrm{E}\left\{E_{b}\right\} \geq \mathrm{E}\left\{E_{c}\right\}$, where $\mathrm{E}\{\cdot\}$ denotes the expectation, $E_{b}$ and $E_{c}$ are defined by (3) and (4).

This proposition evaluates whether the expected harvested energy is greater than the average consumption. Section IV validates this proposition and demonstrates the outage using hardware experiments on PowerCast's power harvester [2].

\section{OUtAGE OF SWIPT SYSTEMS}

This section studies the outage performance of power harvesting and information transmission. Based on these two components, a joint closed-form of SWIPT outage is proposed. We further demonstrate how to obtain the optimal splitting ratio for PS based SWIPT systems using the closed-form. The same method can be used for optimising other parameters of SWIPT systems to achieve minimum outage.

\section{A. Power outage probability}

We first define the power outage of a SWIPT system as follows,

$$
P=\operatorname{Pr}\left\{E_{b}<E_{c}\right\}
$$

which represents the probability that the instant available energy is insufficient for consumption.

The power states of a wireless node can be modelled using a Markov Chain, shown in Fig. 1. Each node may experience one of the following two states at each time slot: sufficient power for information transfer, denoted as $H_{1}$ (the power sufficient state), and insufficient power for

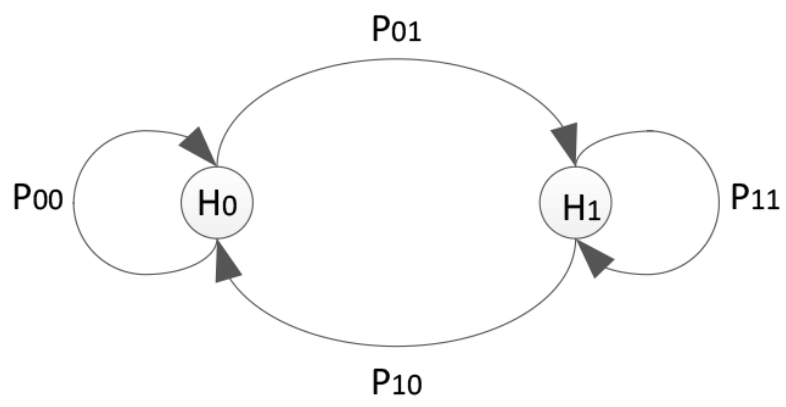

Fig. 1: The Markov Chain model of a wireless node without battery ( $H_{0}$ : Outage, and $H_{1}$ : Sufficient power).

information transfer, denoted as $H_{0}$ (the power outage state).

The transition probabilities between two states $(i$ and $j$ ) are denoted as $P_{i j}$, where $i$ and $j$ are either 0 (to represent $H_{0}$ ) or 1 (to represent $H_{1}$ ). For a chain of power state transitions, we analyse its probability evolution under two different settings: $C=0$ if there is no energy storage unit, and $C>0$ if the node is installed with a rechargeable battery or supercapacitor with a capacity of $C$, which stores energy harvested from both the SWIPT signals and ambient RF signals.

1) No energy storage unit: If $C=0$, the above Markov Chain would reach a steady state as defined by the following equations,

$$
\left\{\begin{array}{l}
\pi_{0} P_{00}+\pi_{1} P_{10}=\pi_{0} \\
\pi_{0} P_{01}+\pi_{1} P_{11}=\pi_{1}
\end{array}\right.
$$

where $\pi_{0}$ and $\pi_{1}$ are the steady-state probabilities of the two states $H_{0}$ and $H_{1}$. It is easy to know that $\pi_{0}+\pi_{1}=1$, $P_{00}+P_{01}=1$ and $P_{10}+P_{11}=1$. Therefore,

$$
\left\{\begin{array}{l}
\pi_{0}=\frac{P_{10}}{P_{01}+P_{10}} \\
\pi_{1}=\frac{P_{01}}{P_{01}+P_{10}}
\end{array}\right.
$$

We can work out the above two equations and get the overall power outage probability as follows,

$$
P_{p, o}=1-\pi_{1}=1-\frac{P_{01}}{P_{01}+P_{10}}
$$

The two steady state probabilities within the above equations denote the sufficient and outage conditions between power harvesting yield and consumption,

$$
\left\{\begin{array}{l}
\pi_{01}=\operatorname{Pr}\left\{E_{b} \geq E_{c}\right\} \\
\pi_{10}=\operatorname{Pr}\left\{E_{b}<E_{c}\right\}
\end{array}\right.
$$

Rician distribution is adopted for channel modelling when there exists the LoS domination path together with small scatters. The Rician factor $s$ defines the ratio between the power from the direct path and the scatters. For the cases that $s$ approaches 0 , the direct path has comparable power as the scatters, and the Rician distribution becomes the Rayleigh distribution. We introduce $g=|h|^{2}$ to denote the channel gain, which follows the non-central exponential 


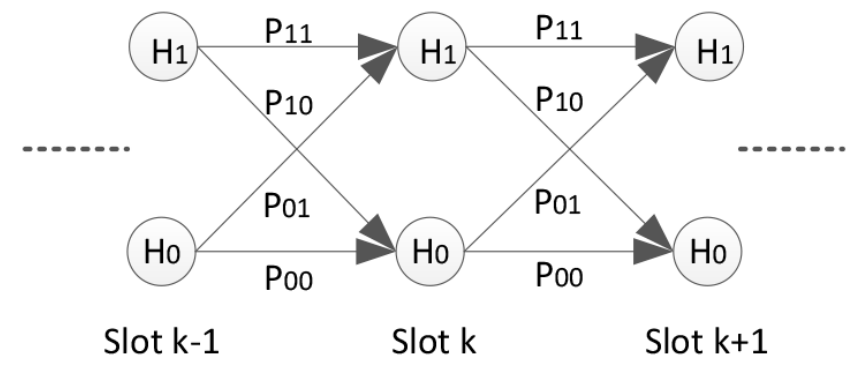

Fig. 2: The Markov Chain model of a wireless node with a rechargeable battery $\left(H_{0}\right.$ : Outage, and $H_{1}$ : Sufficient power).

distribution with a probability density function (PDF): $f_{\lambda}(g)=I_{0}(2 \sqrt{\lambda s g}) \lambda e^{-\lambda g-s}, 0<g<\infty$ [28]. Therefore,

$$
\begin{aligned}
P_{01} & =\operatorname{Pr}\left\{\beta \eta\left(1-\theta_{s}\right) P T_{s}\left(\frac{|h|^{2}}{1+d^{\alpha}}\right) \geq E_{c}\right\} \\
& =\int_{E_{c} / \gamma}^{+\infty} I_{0}(2 \sqrt{\lambda s g}) \lambda e^{-\lambda g-s} \mathrm{~d} g \\
& =\exp \left\{-\frac{\lambda E_{c}}{\gamma}-s\right\} \sum_{n=0}^{+\infty} \frac{s^{n}}{n !} \sum_{m=0}^{n} \frac{\left(\lambda E_{c}\right)^{m}}{\gamma^{m}(m !)}
\end{aligned}
$$

where $\gamma=\frac{\beta \eta\left(1-\theta_{s}\right) P T_{s}}{1+d^{\alpha}} . I_{0}$ is the 0 -th order Bessel function of the first kind, defined as

$$
I_{0}(x)=\sum_{n=0}^{+\infty} \frac{x^{2 n}}{(n !)^{2}}
$$

$P_{10}$ can be similarly obtained as,

$$
P_{10}=1-\exp \left\{-\frac{\lambda E_{c}}{\gamma}-s\right\} \sum_{n=0}^{+\infty} \frac{s^{n}}{n !} \sum_{m=0}^{n} \frac{\left(\lambda E_{c}\right)^{m}}{\gamma^{m}(m !)}
$$

We can thus obtain the steady-state power sufficiency probability as follows

$$
\pi_{1}=\exp \left\{-\frac{\lambda E_{c}}{\gamma}-s\right\} \sum_{n=0}^{+\infty} \frac{s^{n}}{n !} \sum_{m=0}^{n} \frac{\left(\lambda E_{c}\right)^{m}}{\gamma^{m}(m !)}
$$

and the overall outage probability during $K$ continuous time slots as

$$
\begin{aligned}
P_{p, o} & =1-\prod_{j=1}^{K} \pi_{1} \\
& =1-e^{-\lambda \frac{K E_{c}}{\gamma}-K S}\left(\sum_{n=0}^{+\infty} \frac{s^{n}}{n !} \sum_{m=0}^{n} \frac{\left(\lambda E_{c}\right)^{m}}{\gamma^{m}(m !)}\right)^{K} .
\end{aligned}
$$

For the special case of $K=1$, the outage probability can be regarded as the instantaneous power outage performance for each time slot.

2) With an energy storage unit and ambient power harvest: The model is shown in Fig. 2, where the states of a wireless node at the $(k-1)$ th, $k$ th and $(k+1)$ th time slot are displayed. We denote the total number of continuous messages (including the received and transmitted messages) as $K$, and the initial state of the battery is $C$.
Following a similar analysis method as in the previous section, we have the two states $H_{0}$ and $H_{1}$ denoting power sufficient state and outage state, respectively. The transition probabilities from any state at $k$ to the states at $k+1$ are given by $P_{00}(k), P_{01}(k), P_{10}(k)$ and $P_{11}(k)$.

In order to calculate the outage, $P_{11}(k)$, which denotes the probability of staying at an energy sufficient state at time $k$ (i.e., the cumulative energy yield is no less than the consumption), is worked out first,

$$
\begin{aligned}
P_{11}(k) & =\operatorname{Pr}\left\{\sum_{j=1}^{k} \beta \eta\left(1-\theta_{s}\right) P T_{s}\left(\frac{g_{j}+S}{1+d^{\alpha}}\right)+C \geq k E_{c}\right\} \\
& =\operatorname{Pr}\left\{\sum_{j=1}^{k} g_{j} \geq \frac{k E_{c}-C}{\gamma}\right\} .
\end{aligned}
$$

Given each $g_{j} \geq 0$, if $C \geq k E_{c}$, the above probability will always be $100 \%$.

Let $G(k, \lambda)=\sum_{j=1}^{k} g_{j}=x$. Considering the sum of the Rice factors $S=\sum_{j=1}^{k} s_{j}=k s$. The power gain $G(k, \lambda)$ follows non-central $\chi^{2}$ distribution [29] with its PDF as

$$
f(x)=\lambda\left(\frac{\lambda x}{s}\right)^{\frac{k-1}{2}} e^{-\lambda x-k S} I_{k-1}(2 \sqrt{\lambda k S x}) .
$$

We can then obtain the following closed form

$$
\begin{aligned}
P_{11}(k) & =\int_{\frac{k E_{c}-C}{\gamma}}^{\infty} f(x) \mathrm{d} x \\
& =\exp \left(-\lambda \frac{k E_{c}-C}{\gamma}\right) \sum_{n=0}^{+\infty} \frac{(k S)^{n}}{n !} \\
& \cdot \sum_{m=0}^{n+k-1} \frac{\left[\frac{\lambda\left(k E_{c}-C\right)}{\gamma}\right]^{m}}{m !}
\end{aligned}
$$

Based on the Markov model in Fig. 2, the overall outage of the wireless system during $K$ time slots can be expressed as follows

$$
P_{p, o}=1-\prod_{k=1}^{K} P_{11}(k)
$$

A robust wireless system that functions sustainably should achieve as low power outage as possible. In a practical scenario, two power resources could be exploited, for example, power from the dedicated RF sources and ambient RF signals.

Proposition 3.1: The necessary condition to achieve a predefined minimum power outage probability is that the capacity of the power storage unit of a wireless node meets the total power consumption during $K$ continuous functioning time slots: $C=K E_{c}$. At the extreme scenarios, the minimum power outage probability could reach 0 .

Proof: From the Markov chain model, the proof of Proposition 3.1 can be broken into several single steps from 
$k=1$ to $k=K$. Firstly, we take the derivative of $P_{11}(k)$,

$$
\begin{aligned}
& \frac{\partial P_{11}(k)}{\partial C}=\frac{e^{-\lambda \frac{k E_{c}-C}{\gamma}-k S}}{\gamma} \sum_{n=0}^{+\infty} \frac{(k S)^{n}}{n !} \\
& \cdot\left(\sum_{m=0}^{n+k-1} \frac{\left[\frac{\lambda\left(k E_{c}-C\right)}{\gamma}\right]^{m}}{m !}-\sum_{m=1}^{n+k-1} \frac{\left[\frac{\lambda\left(k E_{c}-C\right)}{\gamma}\right]^{m-1}}{(m-1) !}\right) \\
& =\frac{e^{-\lambda \frac{k E_{c}-C}{\gamma}-k S}}{\gamma} \sum_{n=0}^{+\infty} \frac{(k S)^{n}}{n !} \frac{\left(\frac{\lambda\left(k E_{c}-C\right)}{\gamma}\right)^{n+k-1}}{(n+k-1) !}
\end{aligned}
$$

Working out the right hand side of the above equation gives just one term as follows,

$$
\begin{aligned}
\frac{\partial P_{11}(k)}{\partial C}= & \exp \left\{-\lambda \frac{k E_{c}-C}{\gamma}-k S\right\} \\
& \cdot I_{k-1}\left(2 \sqrt{\lambda k S \frac{k E_{c}-C}{\gamma}}\right)\left(\frac{\lambda\left(k E_{c}-C\right)}{\gamma k S}\right)^{\frac{k-1}{2}}
\end{aligned}
$$

By assigning $\frac{\partial P_{p, o}(k)}{\partial C}=0$, we have

$$
C=k E_{c} \text {. }
$$

Combining the conditions for each $k$ from 1 to $K$, and $C \geq k E_{c}$, we have that if the total available energy is $C=$ $K E_{c}$, the power outage probability could reach minimum during $K$ time slots.

This result suggests that, for a theoretically perpetual working wireless node equipped with RF energy harvesting capabilities, the minimum available power and the capacity of the energy storage should be no less than the designed maximum power consumption.

However, most practical systems usually allow outage occuring at a certain probability level, which can be calculated by (17). Another important factor is the average residual power left in the energy storage unit after $K$ time slots, which provides the foundation of the next $K$ time slots, which is given below,

Proposition 3.2: The average power after $K$ continuous time slots is $C_{\text {residual }}=\gamma K / \lambda-K E_{c}+C$.

Proof: The proof of Proposition 3.2 is as follows,

$$
\begin{aligned}
C_{\text {residual }} & =\mathrm{E}\left\{E_{b}-K E_{c}+C\right\} \\
& =\gamma \mathrm{E}\left\{\sum_{j=1}^{K} g_{j}\right\}-K E_{c}+C
\end{aligned}
$$

Incorporation of the probability distribution of $\sum_{j=1}^{K} g_{j}$ gives the closed form, as follows,

$$
C_{\text {residual }}=\gamma K / \lambda-K E_{c}+C .
$$

From Proposition 3.2, a sustainable wireless node becomes possible only if $\gamma / \lambda \geq E_{c}$. Otherwise, the whole system would gradually deteriorate to the state similar to the case of no battery in Section III-A1, leading to significantly higher outage probability.

\section{B. Information outage probability}

The main purpose of energy harvesting is for information exchange. It is thus important to evaluate the probability of information outage under the condition that only partial receiving power can be used for information communications, which can be formulated by the probability that data rate supported by the received SNR falls below the required data rate $R$.

We firstly denote the equivalent SNR at the receiver as follows,

$$
\mathrm{SNR}=\frac{\theta_{s} P|h|^{2}}{\left(1+d^{\alpha}\right) \sigma_{p}^{2}}
$$

The outage probability is thus formulated below,

$$
P_{10}^{\prime}=\operatorname{Pr}\{\log (1+\mathrm{SNR})<R\},
$$

and the steady-state outage probability can be obtained similar to (7),

$$
\pi_{1}^{\prime}=\frac{P_{01}^{\prime}}{P_{01}^{\prime}+P_{10}^{\prime}}
$$

where the probability is taken over all possible $g=|h|^{2}$, given below,

$$
\begin{aligned}
P_{10}^{\prime} & =\int_{0}^{\mu} f_{\lambda}(g) \mathrm{d} g \\
& =1-\exp (-\lambda \mu-S) \sum_{n=0}^{+\infty} \frac{S^{n}}{n !} \sum_{m=0}^{n} \frac{(\lambda \mu)^{m}}{m !}
\end{aligned}
$$

where $\mu=\frac{\left(1+d^{\alpha}\right)\left(2^{R}-1\right)}{\theta_{s} \rho}$, and $P_{01}^{\prime}+P_{00}^{\prime}=1$ and $P_{10}^{\prime}+$ $P_{11}^{\prime}=1$. The steady-state of $H_{1}$ can be obtained as

$$
\pi_{1}^{\prime}=1-P_{10}^{\prime}
$$

After a few mathematical manipulations, we can achieve the closed form of the information outage as follows,

$$
\begin{aligned}
P_{i, o} & =1-\prod_{j=1}^{K} \pi_{1}^{\prime} \\
& =1-\exp (-\lambda K \mu-K S)\left[\sum_{n=0}^{+\infty} \frac{S^{n}}{n !} \sum_{m=0}^{n} \frac{(\lambda \mu)^{m}}{m !}\right]^{K}
\end{aligned}
$$

\section{Joint outage performance}

To measure whether a wireless node can function sustainably, the joint power and information outage can provide a full picture of the outage performance of the system. At any time slot $k$, the joint outage can be expressed as follows,

$$
\begin{aligned}
P_{o}(k) & =1-\left(1-P_{p, o}(k)\right)\left(1-P_{i, o}(k)\right) \\
& =P_{p, o}(k)+P_{i, o}(k)-P_{p, o}(k) P_{i, o}(k)
\end{aligned}
$$

where $P_{p, o}(k)$ is the power outage at time $k$, given by

$$
\begin{aligned}
P_{p, o}(k) & =1-P_{11}(k) \\
& =1-e^{-\lambda \frac{k E_{c}-C}{\gamma}-k S} \sum_{n=0}^{+\infty} \frac{(k S)^{n}}{n !} \sum_{m=0}^{n+k-1} \frac{\left(\lambda \frac{k E_{c}-C}{\gamma}\right)^{m}}{m !}
\end{aligned}
$$


and $P_{i, o}(k)$ is the information outage at time $k$, given by

$$
\begin{aligned}
P_{i, o}(k) & =1-\pi_{1}^{\prime} \\
& =1-\exp (-\lambda \mu-S) \sum_{n=0}^{+\infty} \frac{S^{n}}{n !} \sum_{m=0}^{n} \frac{(\lambda \mu)^{m}}{m !}
\end{aligned}
$$

It is easy to see that, there exists a trade-off between power outage and information outage, which is primarily controlled by the power splitting factor $\theta_{s}$. The following theorem gives the optimal $\theta_{s}$ at the time slot $k$ that leads to the minimum joint outage probability.

Theorem 3.3: The optimal power splitting factor $\theta_{s}$ for a minimum overall outage probability, represented as $\theta_{s}=$ $1-x$, is given by the roots of the following polynomial,

$$
\sum_{n=0}^{k-1} \frac{\epsilon(\xi+x k S)^{n-k+1}(k-1) !}{n !} x^{k+1-n}+(1-x)^{2} \xi=0 \text {. }
$$

Proof: To prove Theorem 3.3, we firstly obtain the derivative of $P_{o}(k)$ against $\theta_{s}$ as follows,

$$
\frac{\partial P_{o}(k)}{\partial \theta_{s}}=\frac{\partial P_{p, o}(k)}{\partial \theta_{s}}\left(1-P_{i, o}\right)+\frac{\partial P_{i, o}(k)}{\partial \theta_{s}}\left(1-P_{p, o}\right),
$$

where

$$
\begin{aligned}
\frac{\partial P_{p, o}(k)}{\partial \theta_{s}}= & e^{-\lambda \frac{k E_{c}-C}{\gamma}-k S} \frac{\gamma^{\prime} \lambda\left(k E_{c}-C\right)}{\gamma^{2}} \\
& \cdot \sum_{n=0}^{+\infty} \frac{(k S)^{n}}{n !} \frac{\left.\left(\lambda \frac{k E_{c}-C}{\gamma}\right)^{(} n+k-1\right)}{(n+k-1) !}
\end{aligned}
$$

and

$$
\gamma^{\prime}=\frac{\partial \gamma}{\partial \theta_{s}}=\frac{-\beta \eta P T_{s}}{1+d^{\alpha}} .
$$

Let $\xi=\frac{\lambda\left(k E_{c}-C\right)\left(1+d^{\alpha}\right)}{\beta \eta P T}$, we have

$$
\begin{aligned}
\frac{\partial P_{p, o}(k)}{\partial \theta_{s}}= & e^{-\frac{\xi}{1-\theta_{s}}-k S} \frac{\xi}{\left(1-\theta_{s}\right)^{2}}\left[\frac{\xi}{k S\left(1-\theta_{s}\right)}\right]^{\frac{k-1}{2}} \\
& \cdot I_{k-1}\left(2 \sqrt{k S \xi /\left(1-\theta_{s}\right)}\right) .
\end{aligned}
$$

The second derivative is achieved as follows,

$$
\begin{aligned}
\frac{\partial P_{i, o}(k)}{\partial \theta_{s}}= & \exp \left\{\frac{\epsilon}{\theta_{s}}\right\} \frac{\epsilon}{\theta_{s}^{2}} \sum_{n=0}^{+\infty} \frac{S^{n}}{n !} \frac{\left(\frac{\epsilon}{\theta_{s}}\right)^{n}}{n !} \\
& =\exp \left\{\frac{\epsilon}{\theta_{s}}\right\} \frac{\epsilon}{\theta_{s}^{2}} I_{0}\left(2 \sqrt{\epsilon /\left(S \theta_{s}\right)}\right),
\end{aligned}
$$

where $\epsilon=\frac{-\lambda\left(1+d^{\alpha}\right)\left(2^{R}-1\right)}{\rho}$. We expand the expression above and obtain the following,

$$
\begin{aligned}
\frac{\partial P_{o}(k)}{\partial \theta_{s}} & =\left\{\frac{e^{-\frac{\xi}{1-\theta_{s}}}-k S}{(k-1) !}\left(\frac{\xi}{1-\theta_{s}}+k S\right)^{k-1} \frac{\xi e^{\frac{\epsilon}{\theta_{s}}}}{(1-\theta)^{2}}\right. \\
& \left.+e^{\frac{\epsilon}{\theta_{s}}} \frac{\epsilon}{\theta_{s}^{2}} \sum_{n=0}^{k-1} \frac{1}{n !} e^{-\frac{\xi}{1-\theta_{s}}-k S}\left(\frac{\xi}{1-\theta_{s}}+k S\right)^{n}\right\} .
\end{aligned}
$$

Assign the above expression with zero and generate the optimal $\theta_{s}$ associated with the minimum overall outage. It is easy to see that the shared exponential terms are greater than 0 , therefore, we can simplify the expression as follows,

$$
\begin{gathered}
\frac{1}{(k-1) !}\left(\frac{\xi}{1-\theta_{s}}+k S\right)^{k-1} \frac{\xi}{(1-\theta)^{2}} \\
+\frac{\epsilon}{\theta_{s}^{2}} \sum_{n=0}^{k-1} \frac{1}{n !}\left(\frac{\xi}{1-\theta_{s}}+k S\right)^{n}=0
\end{gathered}
$$

After a few mathematical manipulations, the following polynomial can be obtained

$$
\sum_{n=0}^{k-1} \frac{\epsilon(\xi+x k S)^{n-k+1}(k-1) !}{n !} x^{k+1-n}+(1-x)^{2} \xi=0
$$

where $x=1-\theta_{s}$.

The values of $x$ and thus $\theta_{s}$ can be easily obtained by calculating the roots of the above single-variable polynomial, where many numerical methods exist. Details are thus neglected in this paper.

\section{EXPERIMENTS}

This section presents extensive experiments to investigate the power and information outage performance of SWIPT systems. The experiments aim to provide insight on how to optimise a sustainable RF energy harvesting node. Most of the parameters are listed in Table I, with a few exceptions that are specifically described in the relevant experiments. The first part of this section investigates the theoretical outage, and the second part provides hardware experiments to validate the sustainability modelling and outage performance at the no battery scenario (the battery scenario is not experimented due to the hardware limit).

\section{A. Numerical experiments}

1) Power outage performance: The first experiment evaluates the instantaneous power outage probability against the splitting factor $\theta_{s}$ covering the full splitting spectrum, when there is no battery. The analysis model is evaluated against simulation experiments where the Rician factor $s=3 d B$, which means the ratio that the direct power and indirect power contributing to the channel gain is approximately 2. Fig. 3 presents the results, which are evaluated at four distance points. The analysis model's outage probabilities closely match with the points obtained at the simulation experiments. Less power splited from the received signal' total power (larger $\theta_{s}$ ) leads to a higher power outage probability. Ideally, in order to achieve improved power outage performance, we may tend to split more power from the received signal, however, the information outage would increase accordingly. A more balanced solution is to equip a rechargeable battery or supercapacitor with ambient power harvesting, which is confirmed by experiments in the next subsection.

The analysis model is also compared with the ideal harvesting scenario when there is no conversion loss (all RF power could be harvested and stored). Fig. 4 gives the results. It is clear that the ideal scenario sets the upper 


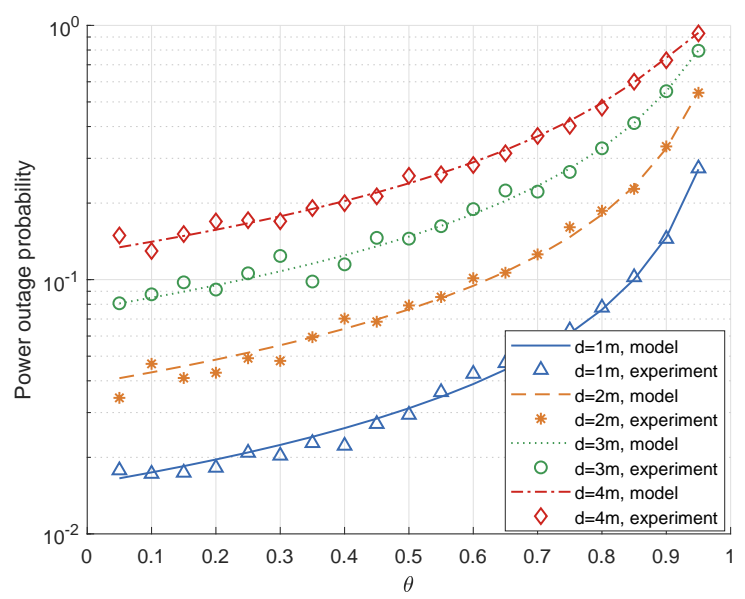

Fig. 3: Power outage probability against power splitting factor in the case of no energy storage unit.

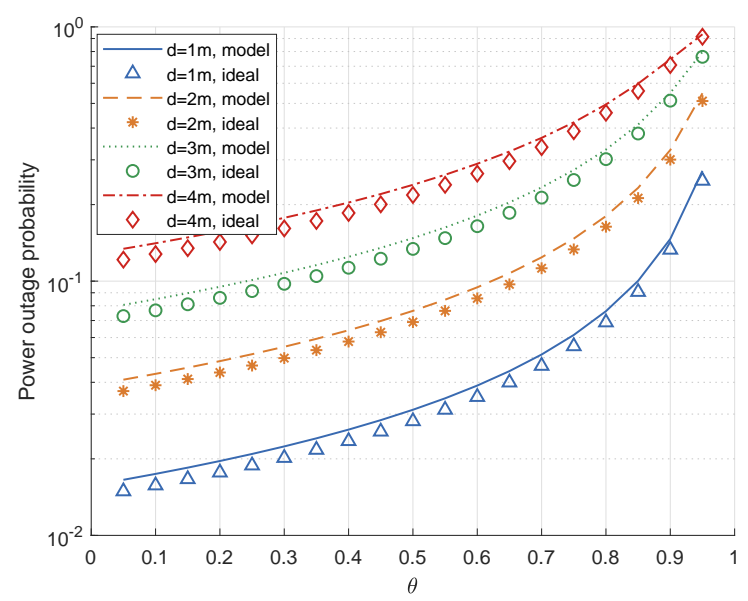

Fig. 4: Power outage probability of the analysis model against optimal benchmarks.

bound for any SWIPT systems. Distance also significantly affects the power outage, for example, when $\theta_{s}=0.5$, the outage probability occurred at $1 \mathrm{~m}$ is approximately $10 \%$ of that of $4 m$.

The next experiment evaluates the analysis model with simulation experiments for the scenario with a power storage unit. The experiment fixes the storage unit to $1500 \mathrm{~nJ}$, and chooses $K$ to $16 . s$ is $3 \mathrm{~dB}$. Fig. 5 presents the results, which are evaluated at four $\theta_{s}$ values. Three observations can be obtained: 1) the analysis model provides accurate performance at the higher outage range, e.g., when they are higher than $10^{-5}$; the approximations introduce errors that become clear when the outage probabilities are low; 2) the power splitting factor $\theta_{s}$ is essential to a satisfactory outage performance: at the same distance, e.g. $d=8 m$, the outage probability for $\theta_{s}=0.8$ is almost $100 \%$, while that for $\theta_{s}$ $=0.2$ is approximately $10^{-5} ; 2$ ) lower distance (especially when $d$ is less than $6 \mathrm{~m}$ ) and higher $\theta_{s}$ are crucial to optimal power outage performance.

To wrap up this subsection, the final experiment studies

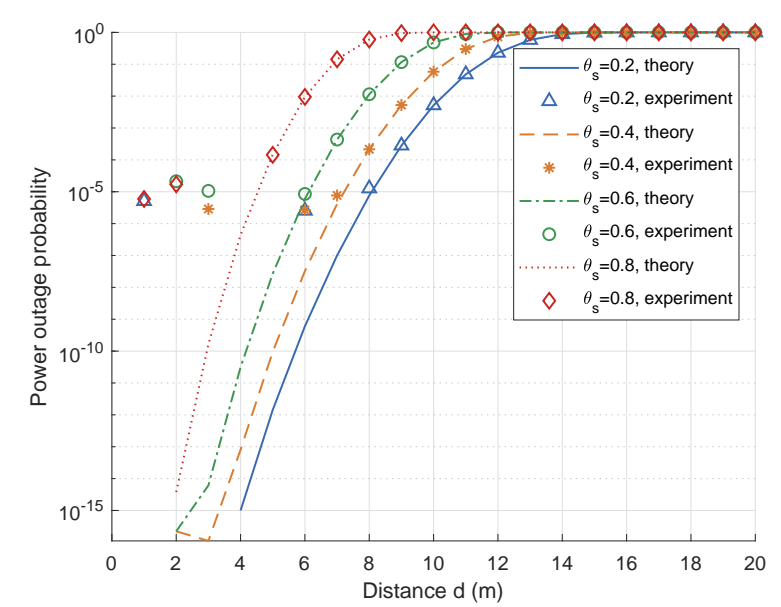

Fig. 5: Power outage probability under varied distance and power splitting factor.

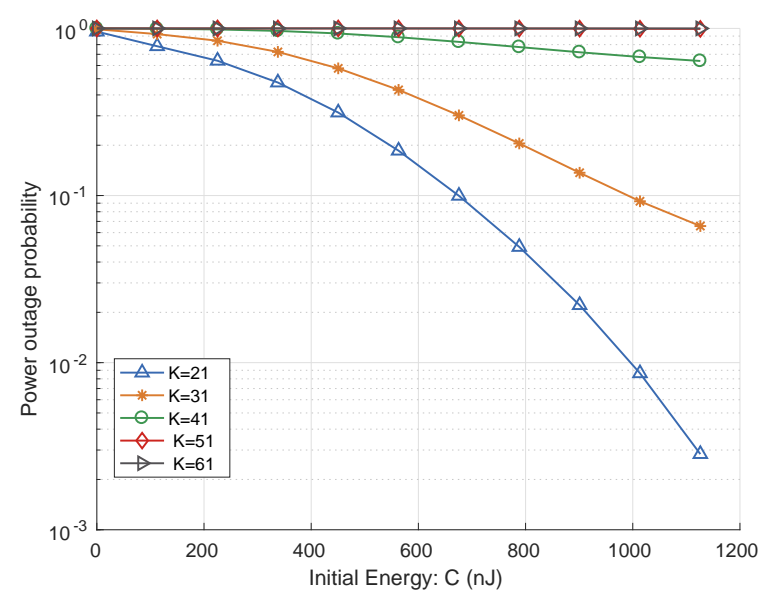

Fig. 6: Continuous power outage probability against ambient power harvest.

the case with varied power available from ambient signals. We fix the distance to $6 m$ and adjust the available power. Fig. 6 illustrates the results, from which we can see the opportunities to harvest energy from ambient signals have a huge impact to the outage of the system. A slight increase in ambient harvesting would make significant contribution to a better power outage performance, particularly when the number of continuous time slots $K$ is small. For example, if the initial power is $1000 \mathrm{~nJ}$, the power outage is $10^{-2}$ after 20 consecutive time slots, which is already better than the best case shown in Fig. 3.

2) Information outage performance: The next group of experiments focus on information outage to measure the probability of exchanging information successfully. Most parameters are also listed in Table I, with a few ones specially explained in each experiment.

Fig. 7 reveals the information outage with regard to SNR and $\theta_{s}$. As expected, the increase in SNR leads to the decrease of information outage. The increase of power share from the received signal also contributes to 


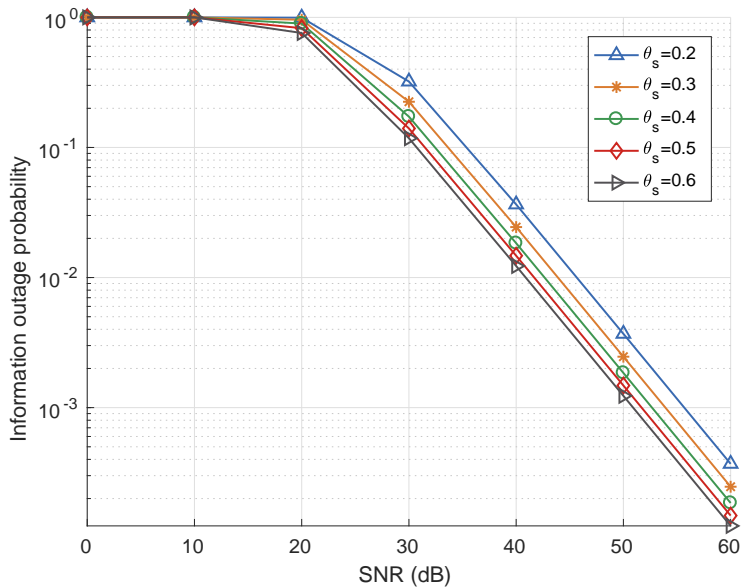

Fig. 7: Information outage probability against SNR.

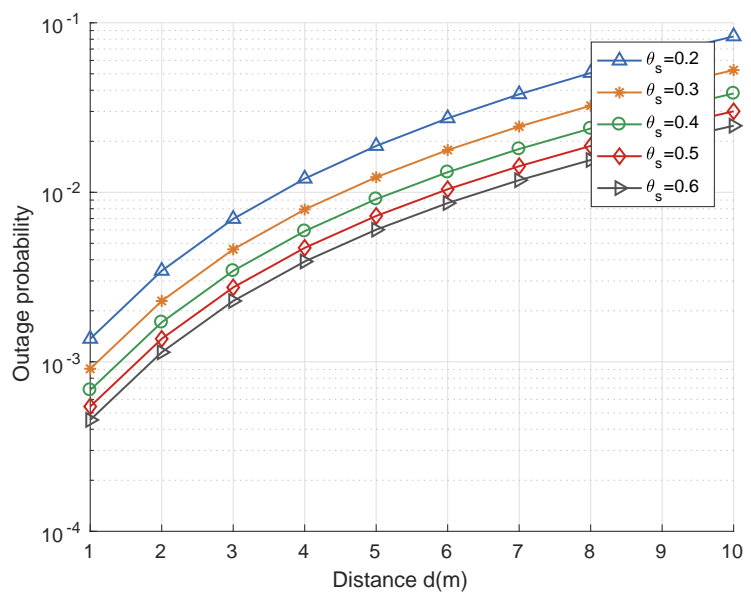

Fig. 8: Information outage probability against distance.

lower outage. The gain becomes less significant when $\theta_{s}$ is greater, e.g. $0.5 \sim 0.6$.

Compared to the power outage in Fig. 5, information outage has a smoother impact on distance changes. Fig. 8 shows some examples when SNR is set to $30 d B$. Generally, smaller distance contributes to lower outage probability, and the increasing tread is similar for different $\theta_{s}$ values.

3) Joint outage and theoretical $\theta_{s}$ : This subsection aims to evaluate the performance of the analysis model of the joint outage, against simulation experiments. The experiment also verifies whether Theorem 3.3 holds or not.

Fig. 9 gives an overall view, where both $K$ and the available power are changed. The distance is chosen to achieve the average power sufficient state $d=3.8 \mathrm{~m}$. The curves represent the joint outage probabilities, while markers represent simulation experiments using Rician channel models. The bowl shapes where the lowest points indicate the place of the ideal $\theta_{s}$. We also use (31) to calculate the theoretical $\theta_{s}$ and the corresponding outage probability of each case, and mark them in the figure using black $\times$ symbols. From the results, we can see Theorem 3.3 holds. The four cases further show that higher power from

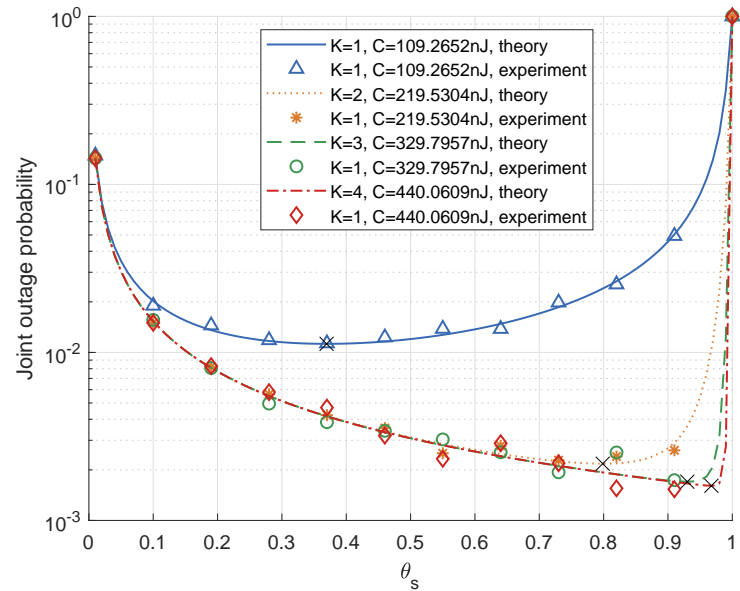

Fig. 9: Joint power and information outage. The symbol $\times$ denotes the theoretical $\theta_{s}$ values that give the minimum outage, calculated using (31).

the storage unit decreases the overall outage probability significantly, and also reveal that more power should be used for information processing if there is sufficient energy in the storage unit.

\section{B. Hardware experiments}

The first hardware experiment investigates the sustainability proposition of SWIPT systems and evaluates the contributions of variable distances between the transmitter and receiver. The hardware and experiment settings are given in Fig.10, in a space without interfering signals at the same band $(915 \mathrm{MHz})$. Spectrum scanner was used to make sure there was no RF signal at the same frequency band, mainly due to the experiment environment being in a new built building without any residents.

The power harvester is P1110, developed by PowerCast and approved by FCC [2]. This experiment simulates the boundary sustainable scenario that all harvested energy is used to power a wireless node without splitting for information transmission.

Fig. 11 presents the experiment results against the theoretical energy consumption model. The results suggest that for the distance less than $7 \mathrm{~m}$ approximately, the average harvested energy is greater than the energy consumption, showing the feasibility of building a sustainable wireless node solely powered by RF signals. Particularly if the distance is less than $1 \mathrm{~m}$, the harvested energy is several times higher than the energy consumption. It is worth mentioning that most of the parameters of the consumption model are adopted from current wireless transceivers and future low-power designs will lower the red dashed curve.

In a practical system, the received signal's power may fluctuate due to channel fading and quite often, the available energy may be insufficient to support communication. The final experiment thus evaluates the analysis model in a laboratory setting. Because the energy receiver (P1110) does not have a power storage unit, this experiment compares the 


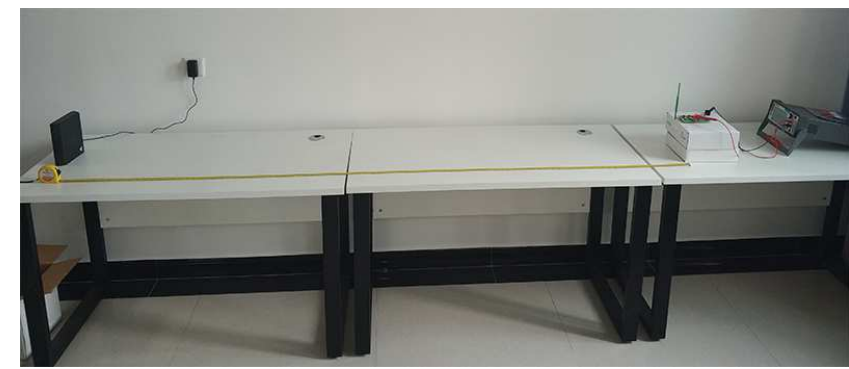

(a) The experiment environment.

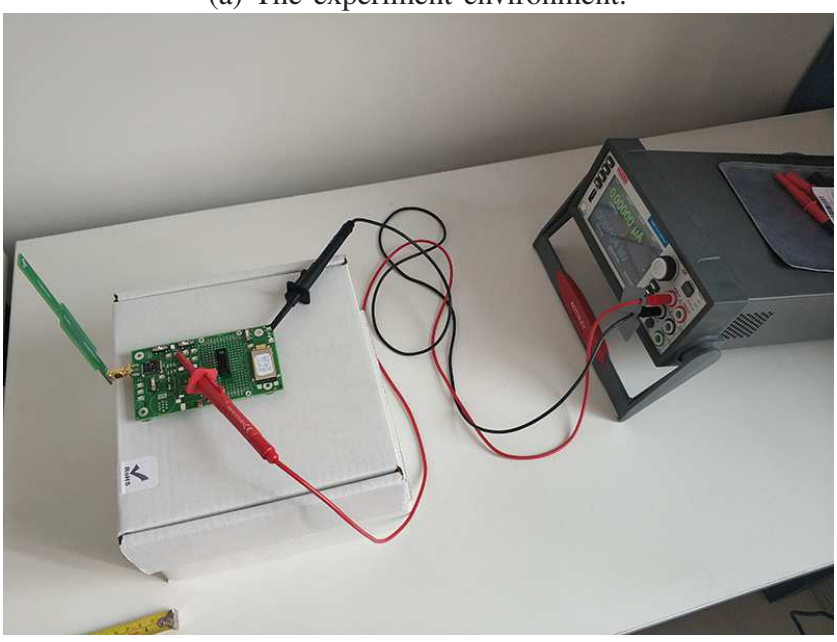

(b) The energy receiver (P1110 Powerharvester Receiver) and measurement meter (Keithley2460).

Fig. 10: Experiment hardware set-up.

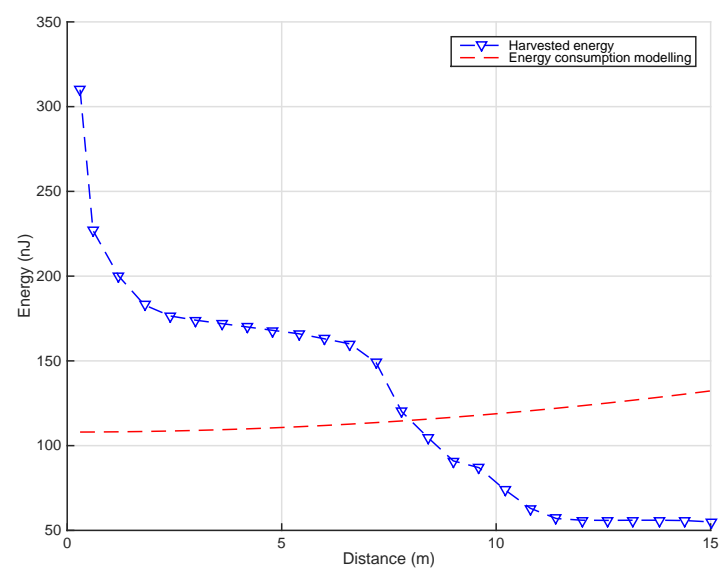

Fig. 11: Harvested energy in experiments and energy consumption model.

performance of the analysis model that that has no battery. 100 experiments at each distance point were collected to calculate the outage performance. Fig. 12 presents the results. Generally, the analysis model provides a close match to the experiment values at a large range of the distance, where measurements at lower outage range are slightly higher than theoretical modelling, showing that the practical environment could not be optimally modelled by Rician channels.

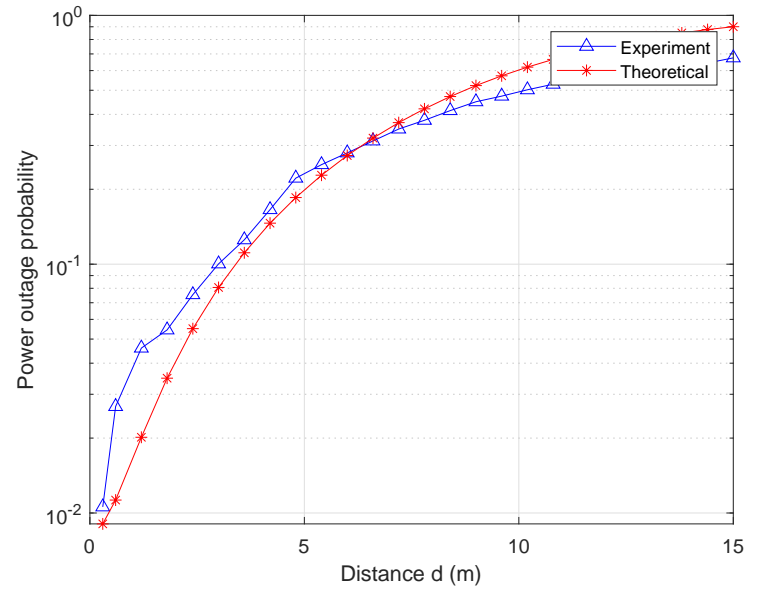

Fig. 12: Outage performance of the analysis model against experiments.

\section{CONCLUSion AND Future Work}

This paper investigates the SWIPT systems' sustainability using both theoretical modelling and practical experiments. We firstly study the energy harvesting and consumption modelling of general wireless systems, and then propose the sustainability condition. We then investigate the power and information outage performance of SWIPT systems and demonstrate how to optimise the SWIPT parameters using power splitting ratio as an example. Hardware and numerical experiments confirm the performance of the proposed models and approaches.

Even though power consumption modelling of wireless nodes has attracted wide research attention, e.g. [25], it will be necessary to investigate contemporary and future lowpower designs, particularly for IoT communications that often require sustainable sensing for substantially long time. This paper uses [25] to establish a common baseline, and does not cover the most up to date models. Our modelling method, however, should still stand for the cases that have different $E_{\text {elec }}$ and $E_{a m p}$.

It would be an interesting topic to investigate the overall network performance in terms of power and information outage in more recent architectures such as relaying and $5 \mathrm{G}$ systems. Some existing work has already touched on this topic, e.g. networking based on energy harvesting [5], relaying models [14], [18], two-way systems [3] etc. The application of the sustainable SWIPT tools studied in this paper could be applied for future study in these fields.

\section{REFERENCES}

[1] H. J. Visser and R. J. M. Vullers, "RF energy harvesting and transport for wireless sensor network applications: Principles and requirements," Proc. IEEE, vol. 101, no. 6, pp. 1410-1423, Jun. 2013.

[2] J. Huang, Y. Zhou, Z. Ning, and H. Gharavi, "Wireless power transfer and energy harvesting: Current status and future prospects," IEEE Wireless Communications, vol. 26, no. 4, pp. 163-169, August 2019.

[3] R. Ma, H. Wu, J. Ou, S. Yang, and Y. Gao, "Power splitting-based swipt systems with full-duplex jamming," IEEE Transactions on Vehicular Technology, vol. 69, no. 9, pp. 9822-9836, 2020. 
[4] X. Chen, D. W. K. Ng, and H. H. Chen, "Secrecy wireless information and power transfer: challenges and opportunities," IEEE Wireless Commun., vol. 23, no. 2, pp. 54-61, Apr. 2016.

[5] K. W. Choi, L. Ginting, A. A. Aziz, D. Setiawan, J. H. Park, S. I. Hwang, D. S. Kang, M. Y. Chung, and D. I. Kim, "Toward realization of long-range wireless-powered sensor networks," IEEE Wireless Communications, vol. 26, no. 4, pp. 184-192, August 2019.

[6] Y. Hu, Y. Zhu, M. C. Gursoy, and A. Schmeink, "Swipt-enabled relaying in iot networks operating with finite blocklength codes," IEEE JSAC, vol. 37, no. 1, pp. 74-88, Jan. 2019.

[7] T. D. Ponnimbaduge Perera, D. N. K. Jayakody, S. K. Sharma, S. Chatzinotas, and $\mathrm{J} . \mathrm{Li}$, "Simultaneous wireless information and power transfer (swipt): Recent advances and future challenges," IEEE Commun. Surveys Tuts., vol. 20, no. 1, pp. 264-302, Firstquarter 2018.

[8] G. Pan, H. Lei, Y. Yuan, and Z. Ding, "Performance analysis and optimization for swipt wireless sensor networks," IEEE Trans. on Commun., vol. 65, no. 5, pp. 2291-2302, May 2017.

[9] Y. Ye, Y. Li, D. Wang, F. Zhou, R. Q. Hu, and H. Zhang, "Optimal transmission schemes for df relaying networks using swipt," IEEE Trans. Veh. Technol., vol. 67, no. 8, pp. 7062-7072, Aug. 2018.

[10] X. Zhou, R. Zhang, and C. K. Ho, "Wireless information and power transfer: Architecture design and rate-energy tradeoff," in 2012 IEEE Global Communications Conference (GLOBECOM), Dec. 2012, pp. 3982-3987.

[11] H. Ju and R. Zhang, "Throughput maximization in wireless powered communication networks," IEEE Trans. Wireless Commun., vol. 13, no. 1, pp. 418-428, Jan. 2014.

[12] R. Zhang, R. G. Maunder, and L. Hanzo, "Wireless information and power transfer: from scientific hypothesis to engineering practice," IEEE Commun. Mag., vol. 53, no. 8, pp. 99-105, Aug. 2015.

[13] X. Lu, I. Flint, D. Niyato, N. Privault, and P. Wang, "Self-sustainable communications with RF energy harvesting: Ginibre point process modeling and analysis," IEEE J. Sel. Areas Commun., vol. 34, no. 5, pp. 1518-1535, May 2016.

[14] M. K. Shukla, H. H. Nguyen, and O. J. Pandey, "Multiuser fullduplex iot networks with wireless-powered relaying: Performance analysis and energy efficiency optimization," IEEE Trans. Green Comm. and Net., vol. 4, no. 4, pp. 982-997, 2020.

[15] J. Xu, L. Liu, and R. Zhang, "Multiuser MISO beamforming for simultaneous wireless information and power transfer," IEEE Trans. Signal Process., vol. 62, no. 18, pp. 4798-4810, Sept. 2014.

[16] M. Sheng, L. Wang, X. Wang, Y. Zhang, C. Xu, and J. Li, "Energy efficient beamforming in MISO heterogeneous cellular networks with wireless information and power transfer," IEEE J. Sel. Areas Commun., vol. 34, no. 4, pp. 954-968, Apr. 2016.

[17] Q. Shi, C. Peng, W. Xu, M. Hong, and Y. Cai, "Energy efficiency optimization for MISO SWIPT systems with zero-forcing beamforming," IEEE Trans. Signal Process., vol. 64, no. 4, pp. 842-854, Feb. 2016.

[18] Y. Li, R. Zhao, Y. Deng, F. Shu, Z. Nie, and A. H. Aghvami, "Harvest-and-opportunistically-relay: Analyses on transmission outage and covertness," IEEE Trans. Wirless Commun., vol. 19, no. 12, pp. 7779-7795, 2020.

[19] S. Peng, T. Wang, and C. Low, "Energy neutral clustering for energy harvesting wireless sensors networks," Ad Hoc Networks, vol. 28, pp. 1 - 16, 2015. [Online]. Available: http: //www.sciencedirect.com/science/article/pii/S1570870515000062

[20] Z. Ding, I. Krikidis, B. Sharif, and H. V. Poor, "Wireless information and power transfer in cooperative networks with spatially random relays," IEEE Trans. Wireless Commun., vol. 13, no. 8, pp. 44404453, Aug. 2014.

[21] R. Zhang and C. K. Ho, "MIMO broadcasting for simultaneous wireless information and power transfer," in 2011 IEEE Global Telecommunications Conference (GLOBECOM), Dec. 2011, pp. 1-5.

[22] Y. Idota, T. Kubota, A. Matsufuji, Y. Maekawa, and T. Miyasaka, "Tin-based amorphous oxide: A high-capacity lithium-ion-storage material," Science, vol. 276, no. 5317, pp. 1395-1397, May 1997.

[23] F.-S. Li, Y.-S. Wu, J. Chou, M. Winter, and N.-L. Wu, "A mechanically robust and highly ion-conductive polymer-blend coating for high-power and long-life lithium-ion battery anodes," Advanced Materials, vol. 27, no. 1, pp. 130-137, 2015. [Online]. Available: http://dx.doi.org/10.1002/adma.201403880

[24] R. Vellacheri, A. Al-Haddad, H. Zhao, W. Wang, C. Wang, and Y. Lei, "High performance supercapacitor for efficient energy storage under extreme environmental temperatures," Nano
Energy, vol. 8, pp. 231 - 237, 2014. [Online]. Available: http: //www.sciencedirect.com/science/article/pii/S2211285514001219

[25] W. R. Heinzelman, A. Chandrakasan, and H. Balakrishnan, "Energyefficient communication protocol for wireless microsensor networks," in Proceedings of the 33rd Annual Hawaii International Conference on System Sciences, 2000, Jan. 2000, pp. 10-21.

[26] I. Sohn, J. H. Lee, and S. H. Lee, "Low-energy adaptive clustering hierarchy using affinity propagation for wireless sensor networks," IEEE Commun. Lett., vol. 20, no. 3, pp. 558-561, Mar. 2016.

[27] Y. Chen, S. Lu, H. S. Kim, D. Blaauw, R. G. Dreslinski, and T. Mudge, "A low power software-defined-radio baseband processor for the internet of things," in 2016 IEEE International Symposium on High Performance Computer Architecture (HPCA), Mar. 2016, pp. $40-51$.

[28] A. Doukas and G. Kalivas, "Rician k factor estimation for wireless communication systems," in 2006 International Conference on Wireless and Mobile Communications (ICWMC'06), July 2006, pp. 69-69.

[29] R. T. Short, "Computation of rice and noncentral chi-squared probabilities," 2012.

[30] Z. Yang, Z. Ding, P. Fan, and G. K. Karagiannidis, "Outage performance of cognitive relay networks with wireless information and power transfer," IEEE Trans. Veh. Technol., vol. 65, no. 5, pp 3828-3833, May 2016. 